\title{
Die Pest in Liestal
}

\author{
Notizen zu den demographischen Implikationen \\ der frühneuzeitlichen Epidemien \\ von Markus Mattmüller
}

Den Jubilar und den Schreibenden verbindet seit Jahren eine fachlich begründete Zusammenarbeit, die sich im Gespräch über die sozialen Auswirkungen der Medizin in vergangenen Epochen angebahnt hat. Den Medizinhistoriker und den Sozialhistoriker interessiert die Seuche der frühen Neuzeit, die Pest; zusammen mit anderen Interessenten haben wir in den Jahren 1975 in Zürich und 1978 in Basel zwei Kolloquien über die schweizerische Pestgeschichte abgehalten, bei denen es zu einer fruchtbaren Begegnung von Medizinern und Historikern kam. In bezug auf dieses gemeinsame Interesse möchte ich einige Mitteilungen zu den demographischen Implikationen der Pestgeschichte machen.

Der Altmeister der schweizerischen Bevölkerungsgeschichte, Wilhelm Bickel, hat die Bevölkerungswelle in unserem Lande auf das Ausbleiben der Pestzüge zurückgeführt ${ }^{1}$. Er vertrat die einfache Hypothese, daß die durchschnittliche Sterblichkeit nach dem Pestzeitalter stark gesunken sei und daß sich aus vorerst noch hoch bleibenden Geburtenziffern in Kombination mit den niedrigen Sterbeziffern jene Beschleunigung des Wachstums ergeben habe, die man seit etwa 1750 wahrnimmt und die erst in allerjüngster Zeit ins Stocken gekommen ist. Bickel erachtete das Aufhören der Pestverluste als Hauptursache und ließ die Schaffung besserer Ernährungsgrundlagen durch Heimindustrie und agrare Reform nur als sekundäre Faktoren gelten.

An sich läßt sich die Bickelsche Hypothese relativ leicht widerlegen, denn es liegt ein zu großes Intervall zwischen dem letzten Pestzug $(1667 / 68)$ und dem Einsetzen der schweizerischen Bevölkerungswelle (um 1750); außerdem sind die großen Wachstumsgebiete der Schweiz im 18. Jh. auch jene, die die letzte Pestwelle noch in voller Stärke erlebt haben, während sich unter den Gebieten schwachen Wachstums solche befinden, die ihre letzte Pest schon um 1630 erlebt hatten. Trotz diesem deutlichen Hinweis auf die Untauglichkeit der Pest-Hypothese soll im folgenden anhand neuerer Forschungsergebnisse noch einmal von den demographischen Folgen der Pest gesprochen werden, denn das Problem ist an und für sich interessant. 
Einer exakten demographischen Erforschung der Peststerblichkeit steht meistens ein Hindernis entgegen: die vollständige Registrierung der Bestattungen in Kirchenbüchern setzte in den meisten Gegenden erst nach dem Aufhören der Pestzüge ein. Für frühere Zeiten findet man bloß Einträge von Taufen und Heiraten, die zwar erlauben, die Erholung einer Bevölkerung nach dem Ende der Seuche zu erfassen, die aber kein Bild vom Bevölkerungsverlust vermitteln. Man kann z. B. die Heiraten und Taufen in den beiden Kirchgemeinden Liestal und Lausen von 1542 bis heute verfolgen, aber auf Grund der registrierten Taufen und Heiraten kann man von den acht Pestzügen, die zwischen 1540 und 1670 durchgezogen sind, nur zwei identifizieren. Ein Tiefpunkt der Hochzeiten in der Pestzeit und eine massive Steigerung nachher, ein Ausfall von Taufen während und kurz nach der Epidemie und ein deutlicher Nachholgipfel finden sich also offensichtlich nicht bei allen Seuchenzügen; sie reichen damit nicht aus zur Identifizierung von Pestjahren.

Glücklicherweise haben in der Kirchgemeinde Liestal-Seltisberg BL gelegentlich die Pfarrer auch Todesfälle registriert, und zwar ziemlich vollständig; das mag seinen Grund gerade in Pestzügen gehabt haben, denn diese gewaltige Sterblichkeit scheint das Interesse von Geistlichen erregt zu haben, so daß sie die Erscheinung auch quantitativ erfassen wollten.

Drei von acht Pestzügen lassen sich so in der Kirchgemeinde Liestal studieren, indem dreimal ein Pfarrer die Sterbefälle registriert hat, 1593/94, 1636 und 1667/68. Der Geschichtsschreiber der Basler Landschaft, K. Gauss, hat eine Reihe von Wortberichten über diese drei Seuchenzüge zusammengestellt ${ }^{2}$; sie erbringen den Beweis, daß es sich bei den im Kirchenbuch feststellbaren Sterbegipfeln wirklich um Pestepidemien gehandelt hat. $\mathrm{Ob}$ alle Opfer registriert worden sind, läßt sich nicht mit Sicherheit feststellen; es ist anzunehmen, daß die Zahl der Opfer höher gelegen hat - die Pfarrer pflegten ja nur jene Verstorbenen einzutragen, die sie selber eingesegnet hatten. Aber es läßt sich doch anhand der Einträge ein Bild von den demographischen Folgen der Seuche gewinnen.

Die Seuche von 1593 erfolgte relativ kurz nach einer Zählung der Wehrfähigen, die im Jahr 1585 durchgeführt worden war und die uns -anhand einer wissenschaftlich verantworteten Hochrechnung - ein Bild von der Einwohnerzahl Liestals vor der Seuche gibt. Die Kleinstadt Liestal und das zur gleichen Kirchgemeinde gehörige Dorf Seltisberg hatten anno 1585 etwa 945 Einwohner $^{3}$.

Anhand der für die ganze Zeitspanne erhaltenen Registrierung von 
Taufen und Bestattungen läßt sich eine «Fortschreibung» der Gesamtbevölkerung versuchen; bei dieser einfachen Operation zählt man einfach zur anfänglichen Einwohnerzahl (945) die Taufen der einzelnen Jahre hinzu und zieht dann die registrierten Bestattungen des gleichen Zeitraums ab; das ergibt folgendes Bild:

Tabelle 1: «Fortschreibungsrechnung» der Bevölkerung der Kirchgemeinde Liestal für die Jahre 1585-1594

\begin{tabular}{|c|c|c|c|c|c|c|c|c|}
\hline \multirow[t]{2}{*}{ Jahr } & \multirow[t]{2}{*}{ Einwohner } & \multicolumn{2}{|c|}{ Taufen } & \multicolumn{2}{|c|}{ Heiraten } & \multicolumn{2}{|c|}{ Bestattungen } & \multirow{2}{*}{$\begin{array}{l}\text { Geburten- } \\
\text { überschuß }\end{array}$} \\
\hline & & $\mathrm{n}$ & Ziffer & $\mathrm{n}$ & Ziffer & n & Ziffer & \\
\hline 1585 & 945 & 47 & 49,4 & 16 & 16,9 & 44 & 46,6 & 3 \\
\hline 1586 & 948 & 47 & 49,6 & 10 & 10,5 & 45 & 47,5 & 2 \\
\hline 1587 & 950 & 40 & 42,1 & 6 & 6,3 & 33 & 34,7 & 7 \\
\hline 1588 & 957 & 4.3 & 44,9 & 11 & 11,5 & 44 & 46,0 & -1 \\
\hline 1589 & 956 & 43 & 45,0 & 4 & 4,2 & 48 & 50,2 & -5 \\
\hline 1590 & 951 & 48 & 50,5 & 12 & 12,6 & 11 & 11,6 & 37 \\
\hline 1591 & 988 & 50 & 50,6 & 12 & 12,1 & 19 & 19,2 & 31 \\
\hline 1592 & 1019 & 52 & 51,0 & 8 & 7,9 & 38 & 37,3 & 14 \\
\hline 1593 & 1033 & 42 & 40,7 & 10 & 9,7 & 78 & 75,5 & -36 \\
\hline 1594 & 997 & 46 & 46,1 & 21 & 21,1 & 203 & 203,6 & -157 \\
\hline 1595 & 840 & & & & & & & \\
\hline
\end{tabular}

Es erhebt sich zunächst die Frage, ob die Registrierung der Bestattungen einigermaßen vollständig ist; im ganzen Ancien Régime findet ja bei der Eintragung der Todesfälle häufig eine Unterregistrierung der Kinder und Säuglinge statt. Die durchschnittliche Sterbeziffer (36,6 Promille) liegt in der Tat im Vergleich mit anderen Regionen etwas tief, aber doch so hoch, daß man nicht eine massive Unterregistrierung befürchten muß. Die Taufziffer (durchschnittlich 47,9 Promille) verdient volles Vertrauen.

Diese «Fortschreibungsrechnung» - die Methode ist derjenigen nachgebildet, welche die Statistiker der heutigen Zeit jeweils im Zeitraum zwischen zwei Volkszählungen anwenden - ermöglicht die Abschätzung des Bevölkerungsverlustes durch die zwei Pestjahre mit ihrer erhöhten Sterblichkeit ${ }^{4}$. Die Bevölkerung hat von 1592 bis 1595 um 179 Seelen abgenommen, also um 17,6\% des Anfangsbestandes. (In Wirklichkeit dürfte der Verlust wegen der Unterregistrierung von Säuglingen und Kleinkindern eher noch etwas höher gelegen haben, etwa bei 20 Prozent.) Man kommt im ersten Jahr der Epidemie auf eine Sterbeziffer von 76 Promille, im zweiten, als die Seuche in 
voller Wucht wütete, auf volle 204 Promille. Erstaunlich ist aber, wie rasch die Bevölkerung die vor der Seuche vorhandene Einwohnergröße wieder erreicht hat. Führt man nämlich die «Fortschreibungsrechnung» weiter bis zu jenem Zeitpunkt, in welchem der Ausgangswert von 1019 Einwohnern wieder erreicht war, so kommt man ins Jahr 1602: Schon nach acht Jahren war der Bevölkerungsverlust rekuperiert!

Die erhaltenen Pfarrbücher ermöglichen es, diese schnelle Regeneration der Bevölkerungsgröße zu analysieren. Die Wachstumsrate im Zeitraum der Rekuperation betrug volle 23,7 Promille pro Jahr; zur Veranschaulichung sei daneben gestellt, daß die durchschnittliche Wachstumsrate in der Kirchgemeinde Liestal zwischen 1585 und 1668 bloß 3,8 Promille betrug. Das heißt, daß der Bevölkerungsverlust durch die Pest einen eigentlichen Wachstumsschub ausgelöst hat. Er kann erklärt werden duch einen hohen Geburtenüberschuß; wir können ihn anhand der Pfarrbücher rekonstruieren.

Die «Fortschreibung» der Bevölkerung nach dem Tiefstand von 1595 zeigt folgendes Bild:

Tabelle 2: Rekuperation in der Kirchgemeinde Liestal 1585-1602

\begin{tabular}{llllllllrl}
\hline Jahr & \multirow{2}{*}{ Einwohner } & \multicolumn{2}{c}{ Taufen } & \multicolumn{2}{c}{ Heiraten } & \multicolumn{2}{c}{ Bestattungen } & \multicolumn{2}{c}{ Geburten- } \\
& & & $\mathbf{n}$ & Ziffer & n & Ziffer & n & Ziffer & überschuß \\
\hline 1595 & 840 & 53 & 61,9 & 22 & 26,2 & 20 & 23,8 & 33 \\
1596 & 872 & 55 & 63,1 & 13 & 14,9 & 30 & 34,4 & 23 \\
1597 & 897 & 45 & 50,2 & 17 & 19,0 & 38 & 42,4 & 7 \\
1598 & 904 & 54 & 59,7 & 13 & 14,4 & 13 & 14,4 & 41 \\
1599 & 945 & 57 & 60,3 & 12 & 12,7 & 25 & 26,5 & 32 \\
1600 & 977 & 56 & 57,3 & 4 & 4,1 & 42 & 43,0 & 14 \\
1601 & 991 & 56 & 56,5 & 17 & 17,2 & 34 & 34,3 & 22 \\
1602 & 1013 & 57 & 56,3 & 12 & 11,8 & 20 & 19,7 & 37 \\
1603 & 1050 & 49 & 46,3 & 16 & 15,2 & 46 & 43,8 & 3 \\
1604 & 1053 & 59 & 56,0 & 16 & 15,2 & 27 & 25,6 & 32 \\
1605 & 1085 & 70 & 64,5 & 16 & 14,7 & 38 & 35,0 & 32 \\
1606 & 1117 & 51 & 45,7 & 23 & 20,5 & 34 & 30,4 & 17 \\
1607 & 1134 & 72 & 63,5 & 18 & 15,9 & 21 & 18,5 & 51 \\
1608 & 1185 & 60 & 50,6 & 9 & 7,6 & 36 & 30,4 & 24 \\
1609 & 1209 & 60 & 49,6 & 12 & 9,9 & 21 & 17,4 & 29 \\
\hline
\end{tabular}

Ein Vergleich der verschiedenen Faktoren, die den Bevölkerungsprozeß bestimmen, zeigt interessante Ergebnisse, wenn man die Zeit vor der Pest 
mit der nach der Pest vergleicht. Die Geburtlichkeit (hier repräsentiert durch die Taufziffer, d.h. Getaufte auf 1000 Einwohner) stellt sich in den acht Jahren vor der Krise (bis 1592) auf den an sich schon hohen Durchschnittswert von 47,8 Promille; in den auf die Seuche folgenden zehn Jahren - bis zur vollständigen Rekuperation - steigt sie auf den fast unglaublichen Wert von 56,8 Promillen ${ }^{5}$. Daß gleichzeitig die Sterblichkeit sinkt (von 36,7 auf 30,6 Promille) kann nicht erstaunen, denn es waren ja in der Pest viele schwache Individuen dahingerafft worden. Es ist also mehr die unerhört hohe Geburtenziffer gewesen, welche ein so starkes Wachstum ermöglicht hat, als eine besonders tiefe Sterblichkeit. In den acht Jahren vor der Pest betrug der durchschnittliche Geburtenüberschuß elf Personen; in den zehn Jahren nach der Pest, der eigentlichen Rekuperationszeit, demgegenüber 24,4 , also mehr als das Doppelte. Und diese Geburtenwelle wurde getragen von einer hohen Nuptialität; sie hatte vor der Pest - wiederum im Durchschnitt von acht Jahren - immerhin schon 10,3 Promille ausgemacht; nach der Pest stieg sie auf 15,1 Promille. Das zeigt das klassische Rekuperationsmodell: Die stark reduzierte Bevölkerung beginnt mit einer Steigerung der Geburtenziffern, die zum Teil durch eine Welle von Heiraten bewirkt wird, zum Teil wohl auch durch höhere Kinderzahlen in den schon länger bestehenden Ehen. Diesen demographischen Entwicklungen entsprechen soziale und ökonomische Realitäten: Die Pest hatte viele Ehen gelöst; die Witwen und Witwer mußten schnell neue Ehen schließen, um die Kinder versorgen zu können; die Pest hatte aber auch viele Ernährungsstellen frei gemacht, Bauernhöfe und Handwerkerstellen waren zu besetzen, so daß nun Menschen einen Arbeitsplatz finden konnten, die sich sonst nur durch Auswanderung oder eine bescheidenere Stelle hätten davonbringen können - und bescheidene Existenzen (landwirtschaftliche Hilfskräfte - «Tauner»und gewerbliche Gesellen) mußten in normalen Zeiten auf die Ehe und damit auf die Fortpflanzung verzichten oder konnten erst spät heiraten.

Die Tatsache einer so raschen Rekuperation der Bevölkerung nach der Pest sagt aber auch etwas über die Zustände vor der Katastrophe. Sie zeigt nämlich, welche Dynamik des Wachstums in einer Bevölkerung steckte und wie starke Bremsmechanismen in normalen Zeiten funktionieren mußten, damit die Bevölkerung nicht zu schnell anwuchs.

Man weiß aus detaillierten Studien, mit welchen Mitteln die alten Gesellschaften das Herauswachsen über den Ernährungsspielraum verhüteten: hohe Ledigenziffern, Spätehe, Auswanderung sind die wichtigsten; daß auch in der Ehe Geburtenkontrolle geübt wurde, ist wahrscheinlich, aber für 
so frühe Zeiten schwer nachzuweisen. Der wesentliche Eingriff einer Pestepidemie in dieses Bevölkerungssystem bestand also darin, daß für kurze Zeit die Kontrolle des Wachstums nicht mehr nötig war und die Bremsmechanismen außer Funktion gesetzt wurden. Die Aufhebung des Eheverzichts für viele Arme wird durch die Zunahme der Heiratshäufigkeit belegt; die Senkung des Heiratsalters läßt sich aus der höheren Taufziffer schließen, denn jüngere Ehepartner sind fruchtbarer. Einzig die Verringerung der Auswanderung können wir weder aus den Pfarr-Registern noch aus anderen Quellen belegen.

Die Registrierung von Todesfällen in Liestal ermöglicht, die Mechanismen der Rekuperation noch an zwei weiteren Pestzügen zu überprüfen. Die nächste größere Epidemie traf Liestal möglicherweise anno 1610/11, aber wir haben für diesen Zeitpunkt keinerlei Registrierung von Bestattungen, so

Tabelle 3: "Fortschreibungsrechnung» der Bevölkerung der Kirchgemeinde Liestal für die Jahre $1635-1656$

\begin{tabular}{|c|c|c|c|c|c|c|c|c|}
\hline \multirow[t]{2}{*}{ Jahr } & \multirow[t]{2}{*}{ Einwohner } & \multicolumn{2}{|c|}{ Taufen } & \multicolumn{2}{|c|}{ Heiraten } & \multicolumn{2}{|c|}{ Bestattungen } & \multirow{2}{*}{$\begin{array}{l}\text { Geburten } \\
\text { überschuf }\end{array}$} \\
\hline & & $\mathrm{n}$ & Ziffer & $\mathrm{n}$ & Ziffer & $\mathrm{n}$ & Ziffer & \\
\hline 1635 & 1144 & 63 & 55,1 & 17 & 14,9 & 83 & 72,6 & -20 \\
\hline 1636 & 1124 & 48 & 42,7 & 4 & 3,6 & 156 & 138,8 & -108 \\
\hline 1637 & 1016 & 64 & 63,0 & 16 & 15,7 & 50 & 49,2 & 14 \\
\hline 1638 & 1030 & 58 & 56,3 & 10 & 9,7 & 87 & 84,5 & -29 \\
\hline 1639 & 1001 & 57 & 57,9 & 21 & 21,0 & 83 & 86,9 & -26 \\
\hline 1640 & 972 & 53 & 54,5 & 9 & 9,3 & 28 & 28,8 & 35 \\
\hline 1641 & 997 & 53 & 53,2 & 15 & 15,0 & 20 & 20,1 & 33 \\
\hline 1642 & 1030 & 58 & 56,3 & 6 & 5,8 & 22 & 21,4 & 36 \\
\hline 1643 & 1066 & 52 & 48,8 & 8 & 7,5 & 23 & 21,6 & 29 \\
\hline 1644 & 1095 & 69 & 63,0 & 9 & 8,2 & 19 & 17,4 & 50 \\
\hline 1645 & 1145 & 50 & 43,7 & 8 & 7,0 & 16 & 14,0 & 34 \\
\hline 1646 & 1179 & 67 & 56,8 & 13 & 11,0 & 40 & 33,9 & 27 \\
\hline 1647 & 1206 & 53 & 43,9 & 17 & 14,1 & 34 & 28,2 & 19 \\
\hline 1648 & 1225 & 65 & 53,1 & 6 & 4,9 & 35 & 28,6 & 30 \\
\hline 1649 & 1255 & 46 & 36,7 & 13 & 10,4 & 18 & 14,3 & 28 \\
\hline 1650 & 1283 & 49 & 38,2 & 10 & 7,8 & 28 & 21,8 & 21 \\
\hline 1651 & 1304 & 49 & 37,6 & 2 & 1,5 & 17 & 13,0 & 32 \\
\hline 1652 & 1336 & 48 & 35,9 & 6 & 4,5 & 31 & 23,2 & 17 \\
\hline 1653 & 1353 & 45 & 33,3 & 10 & 7,4 & 21 & 15,5 & 24 \\
\hline 1654 & 1377 & 56 & 40,7 & 10 & 7,3 & 31 & 22,5 & 25 \\
\hline 1655 & 1402 & 47 & 33,5 & 16 & 11,4 & 17 & 12,1 & 30 \\
\hline 1656 & 1432 & & & & & & & \\
\hline
\end{tabular}


daß wir das Geschehen nur aus nachfolgender Welle von Heiraten ablesen können; dasselbe gilt für die Pestwelle von 1628/29, für welche uns allerdings ein Bericht für Liestal die Zahl von 350 Toten überliefert ${ }^{6}$. Aber als sich derselbe Pestzug anno 1635/36 zurückwandte und wiederum etwa 200 Tote forderte, hat ein Pfarrer die Bestattungen registriert, so daß wir eine «Fortschreibungs-»Rechnung anstellen können.

Man muß sich klar sein, wie groß der Aussagewert einer solchen Tabelle sein kann; schon der Ausgangswert muß ja leider auf einer sehr groben Schätzung beruhen, indem die Einwohnerzahl von 1135 Personen durch grobe Interpolation aus dem entfernten Wert von 1585 hergeleitet werden muß; die erhaltenen Zahlen von Taufen und Bestattungen sind von unterschiedlicher Präzision, weil bei den Bestattungen mit dem Fehlen einiger Kinder und Säuglinge zu rechnen ist. Ferner - und das stellt die größte Quelle für mangelnde Präzision dar - haben wir keinerlei Möglichkeit, die Wanderungsvorgänge abzuschätzen. Aus allen diesen Gründen muß man sich davor hüten, die absolute Höhe der errechneten Ziffern für exakt zu halten; aber der Bevölkerungshistoriker kann aus einer solchen Berechnung doch jenen Vorgang ablesen, der ihn ganz abgesehen von der absoluten Höhe der Ziffern interessiert: das Auf und Ab der vitalstatistischen Verhältnisse nach einem Durchgang der Pest.

Neun Jahre nach der Pest, 1645, war die Einwohnerzahl wieder erreicht, die die Kirchgemeinde vor der Epidemie umfaßt hatte. Die schnelle Rekuperation wurde nicht durch besonders hohe Heiratsziffern bewirkt, sondern durch außerordentlich starke Geburtlichkeit und außerordentlich geringe Sterblichkeit. Es ist also anzunehmen, daß auch Familien, die schon vorher bestanden hatten, nun ungebremster Kinder zeugten. Dreizehn Jahre nach der Seuche, vier nach dem Rekuperationspunkt, sanken die Taufziffern wieder erheblich zurück; die Heiratsziffern überschritten schon im zweiten Jahr nach der Rekuperation ihren letzten Spitzenwert.

Die letzte Pest hat Liestal anno 1668 getroffen, gerade im Jahr nach einer Volkszählung; für einmal müssen wir also in bezug auf den Ausgangswert der «Fortschreibung» keine Bedenken haben.

Zunächst ist zu bemerken, daß die Sterblichkeit bei diesem letzten Pestzug, der die Basler Landschaft getroffen hat, noch weniger hoch stieg als bei der Pest von 1628. Infolgedessen war die Rekuperation bereits im vierten Jahr nach der Seuche vollbracht! Diesmal wirkten allerdings die Heiraten sehr stark mit, um die Lücken aufzufüllen, aber sie fielen dann sogleich nach der vollbrachten Rekuperation auf relativ niedrige Werte zurück. Interes- 
Tabelle 4: «Fortschreibungsrechnung» der Bevölkerung der Kirchgemeinde Liestal für die Jahre $1668-1679$

\begin{tabular}{|c|c|c|c|c|c|c|c|c|}
\hline \multirow[t]{2}{*}{ Jahr } & \multirow[t]{2}{*}{ Einwohner } & \multicolumn{2}{|c|}{ Taufen } & \multicolumn{2}{|c|}{ Heiraten } & \multicolumn{2}{|c|}{ Bestattungen } & \multirow{2}{*}{$\begin{array}{l}\text { Geburten- } \\
\text { überschuß }\end{array}$} \\
\hline & & $\mathrm{n}$ & Ziffer & $\mathrm{n}$ & Ziffer & n & Ziffer & \\
\hline 1668 & 1298 & 62 & 47,8 & 15 & 11,6 & 152 & 117,1 & -90 \\
\hline 1669 & 1208 & 58 & 48,0 & 14 & 11,6 & 25 & 20,7 & 33 \\
\hline 1670 & 1241 & 57 & 45,9 & 19 & 15,3 & 11 & 8,9 & 46 \\
\hline 1671 & 1287 & 65 & 50,5 & 32 & 24,9 & 32 & 24,9 & 33 \\
\hline 1672 & 1320 & 65 & 49,2 & 14 & 10,6 & 39 & 29,5 & 26 \\
\hline 1673 & 1346 & 58 & 43,1 & 6 & 4,5 & 20 & 14,9 & 38 \\
\hline 1674 & 1384 & 75 & 54,2 & 3 & 2,2 & 44 & 31,8 & 31 \\
\hline 1675 & 1415 & 49 & 34,6 & 13 & 9,2 & 59 & 41,7 & -10 \\
\hline 1676 & 14,05 & 51 & 36,3 & 8 & 5,7 & 37 & 26,3 & 14 \\
\hline 1677 & 1419 & 59 & 41,6 & 11 & 7,8 & 41 & 28,9 & 18 \\
\hline 1678 & 1437 & 69 & 48,0 & 18 & 12,5 & 53 & 36,9 & 16 \\
\hline 1679 & 1453 & 67 & 46,1 & 13 & 8,9 & 26 & 13,9 & 41 \\
\hline 1680 & 14.94 & & & & & & & \\
\hline
\end{tabular}

sant ist, daß sich die Geburtlichkeit diesmal, wo wir über einen gesicherten Ausgangswert verfügen, um 50 Promille bewegt und nicht um 55-60 wie in den 1630 er Jahren (das deutet darauf hin, daß die Ziffern in Tabelle 3 durch einen zu niedrigen Ausgangswert überhöht sein könnten); aber auch jetzt sinkt die Taufziffer bald nach der Rekuperation auf recht tiefe Werte zurück, und auch bei dieser Entwicklung treffen wir nach der Pest zunächst eine stark verminderte Sterblichkeit an.

Wenn wir die Reaktion der Bevölkerungsweise auf die drei Pestzüge miteinander vergleichen, so können wir ein generelles Muster feststellen. Hohe Geburtenziffern, die teilweise durch erhöhte Heiratshäufigkeit, teilweise auch durch größere Fruchtbarkeit «alter» Ehen entstanden sein mögen, bewirkten im Zusammenwirken mit einer tieferen Mortalität nach allen drei Pestzügen eine erstaunlich schnelle Rekuperation. War aber der Ausgangswert einmal wieder erreicht, so setzten schnell die alten Bremsmechanismen ein und verhinderten, daß das schnelle Wachstum weiterging. Um mit Malthus zu sprechen, folgte auf eine Periode der «repressive checks» - auf die Pest - und auf die Rekuperationszeit eine Phase, in der die «preventive checks» wieder voll griffen: der in der Bevölkerung vorhandene Trend zum umgebremsten Wachstum wurde durch Erhöhung der Ledigenziffern und durch Geburtenkontrolle innerhalb der Ehen gezähmt ${ }^{7}$. 
Wir kommen damit auf die Ausgangsfrage unserer kurzen Untersuchung zurück. Steht das Aufhören der Pestzüge in irgendeinem Zusammenhang mit der Bevölkerungswelle, welche die Schweiz seit der Mitte des 18. Jahrhunderts ergriffen hat ${ }^{8}$ ? Das Beispiel von Liestal zeigt uns mit voller Deutlichkeit, wie schnell eine Bevölkerung sich auch nach einem starken Aderla $\beta$ durch die Pest regenerierte: In zehn, in neun, ja in vier Jahren erreichte sie wieder den gleichen Stand wie vor der Bevölkerungskatastrophe. Und nachher entwickelte sich die Bevölkerungsweise nicht etwa so, daß diese Dynamik der Rekuperation noch für einige Jahre erhalten blieb, sondern das Geschehen sank relativ schnell wieder ins alte Maß zurück. Damit die Bremsen, die diese traditionellen Gesellschaften gegen ein ungestümes Wachstum eingerichtet hatten, einmal wirklich zu funktionieren aufhörten - und aufhören konnten -, brauchte es andere Anstöße; diese kamen durch eine erhebliche Verbesserung der Erwerbssituation, d.h. durch die Schaffung neuer Arbeitsplätze infolge der Heimindustrie, und das geschah in Liestal in starkem Ausmaße erst in der Mitte des 18. Jahrhunderts. Man ist erstaunt über die Zuverlässigkeit, mit der in einer solchen Gesellschaft des Ancien Régime die doch so vitalen Verhältnisse des Bevölkerungsvorganges reguliert wurden; es regulierte sie eben nicht der menschliche Verstand und die menschliche Einsicht (nach Malthus etwa die «freiwillige Enthaltsamkeit, der «moral restraint»), sondern die pure Notlage, die Knappheit der verfügbaren Ernährungsstellen in Landwirtschaft, Handwerk und Gewerbe. Die gehäuften Todesfälle einer Pestzeit setzten die Regulationsmechanismen nur für kurze Zeit außer Kraft, aber bald nachher rief sich der eiserne Plafond der Subsistenzmittel wieder in Erinnerung.

\section{Anmerkungen}

${ }^{1}$ W. Bickel, Bevölkerungsgeschichte und Bevölkerungspolitik der Schweiz seit dem Ausgang des Mittelalters, Zürich 1947, S. 52.

${ }^{2}$ K.Gauss, Von der Urzeit bis zum Bauernkrieg des Jahres 1653; in: Geschichte der Landschaft Basel und des Kantons Basellandschaft, Bd. I, Liestal 1932, S.506f. und 705 f.

${ }^{3}$ Zahlen und Hochrechnung nach F.Gschwind, Bevölkerungsentwicklung und Wirtschaftsstruktur der Landschaft Basel im 18. Jh., Liestal 1977, S. 115 ff.

${ }^{4}$ Die «Fortschreibungsrechnung» hat einen großen Nachteil, indem sie Wanderungsgewinne und Wanderungsverluste nicht berücksichtigen kann. Wir haben leider keine Quellen, die Wegzug und Zuzug von Personen spiegeln. Unsere Überlegungen basieren also auf dem Modell einer «geschlossenen Bevölkerung», d.h. einer Gesellschaft ohne geographische Mobilität. 
${ }^{5}$ Diese Zahl würde allenfalls etwas nach unten korrigiert, wenn wir die Wanderungsbewegungen miterfassen könnten. Nach einer Pest wanderten gewöhnlich Menschen zu, so daß die Gesamtbevölkerung etwas höher anzusetzen wäre.

${ }^{6}$ K. Gauss, wie Anm. 2, S. 703.

7 Th. R. Malthus, An Essay on the Principle of population (1798), ed. A.Flew, Harmondsworth (Middlesex) 1970, p. 89 und 93.

${ }^{8}$ Vgl. dazu: M. Mattmüller, Das Einsetzen der Bevölkerungswelle in der Schweiz; in: Vierteljahresschrift für Sozial- und Wirtschaftsgeschichte 63.3., Wiesbaden 1976.

\section{Summary}

Parochial registers allow to follow statistically the effects of the plagues of $1593 / 94,1636$, and $1667 / 68$ on the population of a small Swiss town (Liestal). It can be shown that after such a plague the number of marriages and births has grown considerably for a while. Within ten years only population has reached the same number as before, and thereafter remained relatively stable.

Only in the middle of the eighteenth century, due to the beginning industrialisation, better earnings and nutrition caused a population-boom allover Switzerland.

Prof. Dr.phil. Markus Mattmüller

Historisches Seminar

Hirschgäßlein 21

CH-4.051 Basel 